\title{
Clinico-Epidemiological Profile of Malaria
}

\author{
Rajsekhar Patil ${ }^{1}$, Sudheendra Kulkarni ${ }^{2}$ \\ ${ }^{1}$ Department of Medicine, Bidar Institute of Medical Sciences, Bidar, Karnataka, India. \\ ${ }^{2}$ Department of Microbiology, Bidar Institute of Medical Sciences, Bidar, Karnataka, India.
}

\section{ABSTRACT}

\section{BACKGROUND}

Malaria is a major public health problem in India. The clinical manifestations of Malaria range from mild symptoms to severe disease. Death was also observed in many parts of the country. We aimed to study the clinical, epidemiological profile and incidence of Malaria in Bidar district among patients visiting teaching hospital from periphery of the district.

\section{METHODS}

A total of 300 blood samples were collected from cases with history of high fever, chills, profuse sweating, headache, nausea, vomiting, abdominal pain, and muscle pain. Thick and thin smear preparation and examination were carried out.

\section{RESULTS}

Out of 300 samples collected, 13 smears were positive for Malaria (4 cases of $P$. falciparum and 9 cases of $P$. vivax) Majority of subjects were in the age group of 26 35 years, males were affected more compared to females. No statistically significant result was observed.

\section{CONCLUSIONS}

Sporadic pattern of Malaria incidences was observed in the Bidar district. These areas have inadequate sanitation and constant water stagnation, harbouring high vector density and contributing to high infection. Heightened preventive and hygienic measures through educational programmes may substantially reduce the risk of contracting infections in these areas.

\section{KEY WORDS}

Bidar, India, Malaria, NVBDCP, Plasmodium vivax, P. falciparum, Prevalence
Corresponding Author: Dr. Rajsekhar Patil, Associate Professor, Department of Medicine, Bidar Institute of Medical Sciences, Bidar, Karnataka, India. E-mail:drrajsekharpatil@gmail.com

DOI: $10.14260 / j e m d s / 2019 / 753$

Financial or Other Competing Interests: None.

How to Cite This Article: Patil R, Kulkarni S. Clinico-epidemiological profile of malaria. J. Evolution Med. Dent. Sci. 2019;8(46):3479-3481, DOI: 10.14260/jemds/2019/753

Submission 19-09-2019,

Peer Review 28-10-2019,

Acceptance 04-11-2019,

Published 18-11-2019. 


\section{BACKGROUND}

As the second most populous country in the world, with a population exceeding one billion people, Malaria is a major public health problem in India which contributes significantly to the overall malaria burden in Southeast Asia. During recent years 200-300 million clinically suspected cases and half a million deaths were reported worldwide. ${ }^{1}$ Being a vector born disease malaria significantly contributes to the social and economic progress in sub-Saharan Africa, south east Asia and south America. Malaria is a parasitic infection transmitted from person to person by infected Anopheles mosquito. There are four kinds of malaria parasites that can infect humans: Plasmodium vivax, P. ovale, P. malariae, and $P$. falciparum. The two major human malaria species in India are Plasmodium falciparum and P. Vivax..-3 With nearly $95 \%$ of its population residing in malaria-endemic areas, India is battling the burden through implementation of the National Vector Borne Disease Control Program (NVBDCP). ${ }^{4}$ The National Vector Borne Disease Control Program of India reported $\sim 1.6$ million cases and $\sim 1100$ malaria deaths in 2009.5 Historically, the highest incidence of malaria in India occurred in the 1950s, with an estimated 75 million cases and 0.8 million deaths per year (World Health Organization, Country Office for India). Yearly, around 10,000 - 30,000 travellers to endemic region, get infection after returning to their home places. ${ }^{6}$ The states severely affected include Uttar Pradesh, Bihar, Karnataka, Orissa, Rajasthan, Madhya Pradesh and Pondicherry. Malaria is endemic in Karnataka and it has witnessed an increasing incidence of cases over the past 5 years due to increase influx of migrant population. Through continuous efforts of the NVBDCP, malaria is now on a declining trend. ${ }^{6}$ The clinical manifestations of Malaria range from mild symptoms to severe disease and death was also observed in many parts of the country. Vivax malaria usually presents with high grade fever, headache, abdominal pain, nausea and vomiting. Deaths due to malaria occurs by severe anaemia, renal failure, acute respiratory failure, hypoglycaemia, shock, and/or central nervous system involvement (Falciparum malaria).7-8 This study was undertaken to find out the clinical and epidemiological pattern of disease in the Bidar district.

Hence, we aimed to study the clinical, epidemiological profile and incidence of Malaria in Bidar district from patients visiting teaching hospital from periphery of the district.

\section{METHODS}

This cross-sectional study was conducted from August 2018 to February 2019 in the Department of Medicine of 750 bedded Teaching hospital of Bidar Institute of Medical Sciences (BRIMS) Bidar of North Karnataka. The study population consisted patients from urban and rural area of Bidar visiting to Outpatient Department of Medicine complaining of acute fever not less than three days with no evident focus of infection. A total of 300 patients of all age groups and sex visiting to Outpatient department of Medicine complaining of high fever, chills, profuse sweating, headache, nausea, vomiting, abdominal pain, and muscle pain are included in the study. Brief history about the illness and patient details like age, sex and address are recorded. After thorough general physical examination, the findings were entered on a standard data collection sheet. This study protocol was approved by Institute's Ethics Committee, and samples were collected after obtaining informed consent from the patients.

The sample size $(n=300)$ was estimated with an expected prevalence of Malaria as $15 \%$ with $4 \%$ absolute precision and $95 \%$ confidence interval. An interim analysis was carried out and the estimate from the interim analysis was used to modify the sample size. Convenience sampling method was adapted to carry out this study. Blood samples were collected from the patients and sent to the laboratory to carry out complete blood count analysis and thick and thin smear examination for malarial parasites. Thick and thin smears were made from capillary blood were stained with Leishman's stain and observed under oil immersion. Malarial parasite and its spp was identified by microscopy. Smears were repeated every 8 to $12 \mathrm{hrs}$ for the next 48 hours in suspected cases when the first smear did not reveal parasite.

\section{Statistical Analysis}

Statistical software package SPSS version 22 (IBM Corp. Released 2013. IBM SPSS Statistics for Windows, Version 22.0. Armonk, NY: IBM Corp) was used to analyse the data. Chi-square test was applied wherever necessary and P-value of $<0.05$ was considered statistically significant.

\section{RESULTS}

Out of 300 samples collected, 13 smears were positive for Malaria disease. Amongst these 13 cases, 4 cases proved for $P$. falciparum infection and remaining 9 cases were positive for $P$. vivax infection. The most common symptom was pyrexia (All cases) followed by nausea with vomiting, headache. In 4 P. falciparum infection cases the most common complications observed was jaundice followed by cerebral malaria, renal impairment, hypoglycaemia, hepato-renal syndrome. Majority of subjects were in the age group of $26-35$ years, males were affected more compared to females. There was no statistically significant result seen with respect to age and sex. Sporadic pattern of malaria was observed in the Bidar district. Total White blood cell count was normal in majority of affected population and Leukopenia was observed in very less number of cases.

\begin{tabular}{|c|c|c|c|c|}
\hline \multirow{2}{*}{ Characteristics } & \multicolumn{2}{|c|}{ Patients } & \multicolumn{2}{c|}{ Percentage (\%) } \\
\cline { 2 - 5 } & Suspected & Positive & Suspected & Positive \\
\hline Male & 185 & 3 & 61.6 & 75 \\
\hline Female & 115 & 1 & 38.4 & 25 \\
\hline \multicolumn{2}{|c|}{ Table 1. Characteristics of 4 Falciparum Malaria Patients }
\end{tabular}

\begin{tabular}{|c|c|c|c|c|}
\hline \multirow{2}{*}{ Characteristics } & \multicolumn{2}{|c|}{ Patients } & \multicolumn{2}{c|}{ Percentage } \\
\cline { 2 - 5 } & Suspected & Positive & Suspected & Positive \\
\hline Male & 185 & 6 & 61.6 & 66.6 \\
\hline Female & 115 & 3 & 38.4 & 33.4 \\
\hline \multicolumn{3}{|c|}{ Table 2. Characteristics of 9 Vivax Malaria Patients } \\
\hline
\end{tabular}




\section{DISCUSSION}

In the present study 300 patients were studied. Out of which, 4 falciparum malaria and 9 vivax malaria confirmed cases were found which was predominant in this region. In case of vivax malaria infection, males were more affected than females. Also, falciparum malaria cases were more in males compared to females. This may be due to outdoor activities and mobility of males and females are better clothed than males. This observation is consistent with few other studies. Majority of confirmed cases were belonging to age group between 26-35 years fallowed by other age groups. This is consistent with the observation that malaria incidence is higher in young adults because of their outdoor activities and mobility. ${ }^{9-11}$

All patients presented with intermittent/continuous fever. This observation is consistent with few other studies which suggest that fever with chills and rigor is the commonest mode of presentation. Headache was the second commonest symptom similar to the study previously reported.11-12 The classical triad of Malaria was observed in the present study with fever, splenomegaly and anaemia were the commonest findings. Anaemia was reported in majority of cases. Approximately $91 \%$ of confirmed cases had anaemia.

About $65 \%$ of affected population belonged to the middle socioeconomic group and $14 \%$ belonged to lower socioeconomic group. The incidence was lower in upper socioeconomic group. Maximum numbers of malaria cases were observed in monsoon and post monsoon period (August to November). The actual reason behind this is increased breeding in this tenure. This finding is consistent with few other studies. Total WBC count was well within the range in majority of patients. Leukopenia was observed in very less number of population. ${ }^{11-12}$

\section{CONCLUSIONS}

Malaria is prevalent in Bidar and persists throughout the year with maximum level of infection cases seen during the rainy season. Both $P$. vivax and $P$. falciparum infections occur throughout the year. Therefore, conducting educational programmes on malaria awareness and implementation of public control measures are likely to eradicate infection in Bidar.

\section{REFERENCES}

[1] WHO. World malaria report 2015. Geneva: World Health Organization.

http://www.who.int/malaria/publications/worldmalaria-report-2015/report/en/.

[2] Sachs J, Malaney P. The economic and social burden of malaria. Nature 2002;415(6872):680-5.

[3] Hay SI, Okiro EA, Gething PW, et al. Estimating the global clinical burden of Plasmodium falciparum malaria in 2007. PLoS Med 2010;7(6):e1000290.

[4] Kothari A, Pruthi A, Chugh TD. The burden of enteric fever. J Infect Dev Ctries 2008;2(4):253-9.

[5] National Vector borne Disease control Programme. (Last Updated: 15 Dec, 2014; cited on 16 Dec 2014. Assessed on 13 Aug, 2018. http://nvbdcp.gov.in/malarianew.html

[6] World Health Organization, 2007. http://www.whoindia.org/LinkFiles/Malaria. Country Profile-Malaria.pdf.

[7] Sharma VP. Current scenario of malaria in India. Parasitologia 1999;41(1-3):349-53.

[8] Rolling back malaria - the next ten years. Lancet 2008;372(9645):1193.

[9] Murthy GL, Sahay RK, Srinivasan VR, et al. Clinical Profile of falciparum malaria in tertiary care hospital. Journal of Indian Medical Association 2000;98(4):162-9.

[10] Shukla MM, Singh N, Singh MP, et al. Cerebral malaria in Jabalpur, India. Indian Journal of Malariology 1995;32(2):70-5.

[11] Ravishankar MS, Mohan ME, Ramesh TP, et al. Spectral presentation of Plasmodium falciparum malaria in rural Karnataka (Southern India). Biomedical Research 2015;26(3):561-6.

[12] Mehta SR, Naidu G, Chandar V, et al. Falciparum malaria: present day problems. An experience with 425 cases. J Assoc Phys India 1989;37(4):264-7. 